\title{
Symmetry Partitioning over Liouville Space for Evolution under the Anisotropic Quadrupolar Spin-Spin Interaction*
}

\author{
F. P. Temme and B. C. Sanctuary \\ Department of Chemistry, McGill University, Montreal, PQ, Canada** \\ Z. Naturforsch. 41 a, 361-365 (1985); revised version received September 30, 1985
}

\begin{abstract}
The structure of the anisotropic indirect quadrupolar spin-spin interaction, and its effect on the spin dynamics associated with pure NQR time-domain response, is considered in terms of spherical tensor operator bases and multipole theory. The advantages of symmetry-adapting the Liouville bases is discussed for $I \geqq 3 / 2$ spin systems.

In particular, symmetrized state space bases for the $n$-fold quadrupole cluster under $S_{n}$ are introduced and enumerated for $I=3 / 2$ and $n \leqq 4$, as an initial step in the process. Symmetrized Liouville space bases for the $[\mathrm{A}]_{2}$ problems involving [3/2:3/2] and [5/2:5/2] spin pairs under the group $\mathrm{S}_{2}$ are presented to illustrate the structure of the multipole calculations of the spin evolution under the specific spin-spin interaction term. Such systems are of especial interest on account of the nature of the molecular solids $\mathrm{Br}_{2}$ and $\mathrm{I}_{2}$.
\end{abstract}

\section{Introduction}

There has been some renewal of interest in quadrupolar spin-spin, $\mathrm{Q}-\mathrm{Q}$, interactions both in the pure NQR of molecular solids, and as the dominant term for heavy nuclei in zero field NMR [1]. In connection with the former, time-domain techniques hold definite advantages over cw methods [2]. Pulse methods can be conveniently described for all $I$ spins in terms of spherical tensor $T^{k q}(v)$ operator bases [3] and the multipole theory. The $[\mathrm{A}]_{2} \equiv \mathrm{AA}^{\prime}, I_{\mathrm{A}} \geqq 1$ spin system of NMR is amenable to symmetry adaptation under the symmetric group, $S_{2}$ [4]; these developments in respect of Liouville space incorporate many of the ideas used in applying group theory to ordinary state space problems [5]. The inner direct product subgroup [6] retains a significant role.

The observation of $\mathrm{Q}-\mathrm{Q}$ interactions in $\mathrm{NQR}$, from the earliest experiments [7] on molecular $\mathrm{I}_{2}$ to the most recent one on $\mathrm{GaSe}$ [8], have been hindered by inadequate resolution; other experimental methods [9] have offered no improvement on this question. However, such $\mathrm{Q}-\mathrm{Q}$ interactions, for solids involving high spin heavy nuclei, may play an

* Presented at the VIIIth International Syposium on Nuclear Quadrupole Resonance Spectroscopy, Darmstadt, July 22-26, 1985 .

** 801 Sherbrooke St. W., Montreal, PQ, H3A2K6, Canada. Reprint requests to Dr. F. P. Temme, Department of Chemistry, McGill University, 801 Sherbrooke St. W., Montreal, PQ, H3A 2K6, Canada. important role in finally determining whether higher order terms are involved.

The wealth of contributing terms in molecular paramagnetism of metal-metal cluster solids $[10,11]$ has lead to some insight concerning spin-spin interactions for $I \geqq 1$ in NMR [1] with some relevance to NQR. The effective range of $J_{i j}$ and $K_{i j}$ couplings could account for greater structure, only part of which (see eq. (2)) may be observable in NQR at present. Such ideas will be considered in the context of the use of the Liouville equation for spin evolution in a symmetry partitioned form [4], appropriate to spin systems involving permutation symmetry. The spin dynamics of single quadrupolar spins has been established $[12,13]$ and is subject to partitioning of the Liouville equation under the $\mathrm{SO}(3)$ group only in the case of vanishing asymmetry parameters. The $\mathrm{Q}-\mathrm{Q}$ problem incorporates terms from the single spin treatments and then considers the interaction term for $z$ (e.f.g.) taken parallel to $J_{z}$. In addition to any internal permutation symmetry effects, it is necessary to consider the external symmetry of the nuclei in the solid.

The interaction term for like-resonant pairs of spins $[3 / 2: 3 / 2]$, or $[5 / 2: 5 / 2]$ is a weak perturbation, compared to the zero order single quadrupole terms. It may be considered in a coupled tensor form involving spin cluster interactions, as discussed for NMR by Sanctuary and Selwyn [14]. Interhalogen solids could possibly provides examples in NQR of such $(\otimes 3 / 2)_{n}$ clusters under various $\mathrm{S}_{n}$ internal symmetries [4]. Symmetry adaptation of

0340-4811 / $86 / 0100-0361 \$ 01.30 / 0$. - Please order a reprint rather than making your own copy. 
tensor formulations of other forms of spectroscopy have been the subject of much recent theoretical work [15]. The distinction between these uses and symmetry adaptation arising from cluster spin permutation properties in NMR, or NQR, are implicit in the internal nature of the latter and in the totally different bases involved in the two problems.

\section{Theoretical Background}

In the molecular solids, $\mathrm{Cl}_{2}, \mathrm{Br}_{2}$, or $\mathrm{I}_{2}$ the presence of external symmetry derived from the unit cell introduces parameters associated with the mirror plane symmetry. These are analogous to $\mathrm{u} / \mathrm{g}$ designations of certain molecular point groups. For such solids this leads to an additional summation, generally over a pair of molecules related by mirror symmetry.

\subsection{Hamiltonian for Identical Resonant Quadrupoles}

For convenience, the hamiltonian is divided into zeroth order terms and weak perturbations. The form of $H^{(0)}$ adopted needs to reflect the permutation symmetry under a $\mathrm{S}_{n}$ group associated with the quadrupolar spin cluster within the molecule. For the $\mathrm{I}_{2}$ solid previously referred to, this leads to the formulation of the zeroth order hamiltonian as

$H^{(0)}=\sum_{i=1}^{2}-A\left\{3 I_{z}^{2}-I(I+1)\right\}_{i}+\sqrt{3} / 6 \eta\left\{I_{+}^{2}+I_{-}^{2}\right\}_{i}$.

The most general form of the weak perturbation $\mathrm{Q}-\mathrm{Q}$ term is simply

$$
\begin{aligned}
H^{(1)} & =\sum_{i<j}\left\{J_{i j} I_{z i} I_{z j}+K_{i j}\left(I_{x i} I_{x j}+I_{y i} I_{y j}\right)\right\} \\
& =\sum_{i<j}\left\{\left(J_{i j}-K_{i j}\right) I_{z i} I_{z j}+K_{i j} I_{i} \cdot I_{j}\right\},
\end{aligned}
$$

where the last term of (3) corresponds to the bilinear tensor $T^{00}(11)$ of [16]. The form of $J_{i j} I_{z i} I_{z j}$ has more involved form than the dot product under $\mathrm{SO}(3)$, as discussed in the previous paper.

For the $\mathrm{X}_{2}$ halogen solids, (1) leads to a zeroth term for the molecular clusters of

$$
f^{(0)}=\left[\sum_{i i^{\prime}}\left\{-A^{\prime} y_{i}^{20}+\eta / \sqrt{6}\left(y_{i}^{22}+y_{i}^{2-2}\right)\right\},\right]_{-}
$$

with the $i$ and $i^{\prime}$ parameters running over the spins within a cluster, and the clusters within a pair of molecules under the external symmetry. As in the single quadrupole case,

$$
A^{\prime}=e Q q^{2} / 4 \sqrt{(2 I+3)(I+1) /[5 I(2 I-1)]} .
$$

The exact solution of the single spin $5 / 2$ case has already been given in a related paper [13].

The introduction of bilinear Liouville operators for the form of Q-Q interaction suggests the need for a uniform notation. This is possible from the property

$$
\begin{aligned}
& T^{k q}\left(k_{1} k_{2}\right)=\left[\left(2 I_{1}+1\right)\left(2 I_{2}+1\right)\right]^{-1 / 2} \\
& \cdot \sum_{q_{1} q_{2}}(-1)^{k_{1}-k_{2}+k}(-1)^{k-q}\left(\begin{array}{rrr}
k & k_{1} & k_{2} \\
-q & q_{1} & q_{2}
\end{array}\right), y k_{1} q_{1} y k_{2} q_{2}
\end{aligned}
$$

which allows $f^{(0)}$ to be written in the forms $T^{2 q}(20)$ and $T^{2 q}(02)$. The anisotropic interaction then becomes

$$
\begin{aligned}
\mathscr{f}^{(1)}= & \sum \frac{\sqrt{3}}{2} K_{i j} \mathscr{f}^{00}(11) \\
& +\left(J_{i j}-K_{i j}\right)\left\{\mathscr{f}^{20}(11)-\frac{1}{2} \mathcal{f}^{00}(11)\right\}
\end{aligned}
$$

with

$$
\mathcal{f}^{k 0}\left(k_{1} k_{1}\right)=\left[T^{k 0}\left(k_{1} k_{1}\right),\right]_{-} .
$$

Previous evaluations of the isotropic scalar coupling tensor over Liouville space mostly have been limited to the spin $[1 / 2: 1 / 2]$ case.

The coupled tensor format also necessitates a description of the density operator in corresponding terms, namely,

$\sigma(t)=\left\{E+\sum_{k=1}^{2 I_{1}+2 I_{2}^{\prime}} \sum_{q}^{k} \sum_{k_{1} k_{2}} \Phi_{q}^{k}\left(k_{1} k_{2}\right) T^{k q}\left(k_{1} k_{2}\right)\right\}$

whose adjoint, and expectation value (as derived from the trace metric) are fully described in [14]. It follows that the quantum-Liouville equation becomes a set of coupled differential equations

$$
i \hbar \dot{\boldsymbol{\Phi}}=\mathfrak{\rho} \boldsymbol{\Phi}
$$

which span a vector derived from $\boldsymbol{\Phi}$ over the allowed range of parameters $\left(k q: k_{1} k_{2} ; k^{\prime} q^{\prime}: k_{1}^{\prime} k_{2}^{\prime}\right)$. Naturally, partitioning of $\boldsymbol{\Phi}$ into disjoint subspaces as a result of spin permutation symmetry introduces an addition facet to the problem; this is discussed in Section 3.

\subsection{Structure of $\mathcal{L}$ over $\Phi$}

The matrix elements of the $f$ matrix for the single quadrupole spins are already known from 
$[13,16]$ being derived from

$$
《 T^{k q}\left(k_{1} k_{2}\right) \mid\left[T^{2 q}(20), T^{k^{\prime} q^{\prime}}\left(k_{1}^{\prime} k_{2}^{\prime}\right) \quad\right]-》
$$

type terms that are characterized by a $\Phi\left(k l k^{\prime}\right)$ term that vanishes unless $\left(k+l+k^{\prime}\right)$ is odd. All these follows from the Wigner-Eckert theorem which also determines the matrix elements of the bilinear term,

$$
\begin{aligned}
& 《 T^{k q}\left(k_{1} k_{2}\right) \mid\left[T^{l 0}\left(l_{1} l_{2}\right), T^{k^{\prime} q^{\prime}}\left(k_{1}^{\prime} k_{2}^{\prime}\right) \quad\right]_{-} 》 \\
& =2[\quad]^{1 / 2}(-1)^{k-q}\left(\begin{array}{rrr}
k & l & k^{\prime} \\
-q & 0 & q^{\prime}
\end{array}\right)(-1)^{\bar{\mu}}(i)^{\overline{\bar{\mu}}} \\
& \left\{\begin{array}{lll}
k & l_{1} & k^{\prime} \\
I_{1} & I_{1} & I_{1}
\end{array}\right\}\left\{\begin{array}{lll}
k & l_{2} & k^{\prime} \\
I_{2} & I_{2} & I_{2}
\end{array}\right\}\left\{\begin{array}{lll}
l & k^{\prime} & k \\
l_{1} & k_{1}^{\prime} & k_{1} \\
l_{2} & k_{2}^{\prime} & k_{2}
\end{array}\right\} \\
& {\left[\Phi\left(k_{1} l_{1} k_{1}^{\prime}\right)+(-1)^{k_{1}+l_{1}+k_{1}^{\prime}} \Phi\left(k_{2} l_{2} k_{2}^{\prime}\right),\right.}
\end{aligned}
$$

where the phase terms from.(A7) of [16] are

$$
\begin{aligned}
& \bar{\mu}=\left(l+k+k^{\prime}+2\left(I_{1}+I_{2}\right),\right. \\
& \overline{\bar{\mu}}=l_{1}+k_{1}+k_{1}^{\prime}+k_{2}+k_{2}^{\prime}+l_{2} .
\end{aligned}
$$

Restricting our attention to the $[3 / 2: 3 / 2]$ problem and the explicit conventional terms of (3) ensures that (10) becomes

$$
\begin{aligned}
《 & T^{k q}\left(k_{1} k_{2}\right)\left|f^{10}(11)\right| T^{k^{\prime} q^{\prime}}\left(k_{1}^{\prime} k_{2}^{\prime}\right) 》 \\
= & 2[]^{1 / 2}(-1)^{k-q}\left(\begin{array}{rrr}
k & l & k^{\prime} \\
-q & 0 & q^{\prime}
\end{array}\right)\left\{\begin{array}{ccc}
k_{1} & l_{1} & k_{1}^{\prime} \\
3 / 2 & 3 / 2 & 3 / 2
\end{array}\right\} \\
& \cdot\left\{\begin{array}{ccc}
k_{2} & l_{1} & k_{2}^{\prime} \\
3 / 2 & 3 / 2 & 3 / 2
\end{array}\right\}\left\{\begin{array}{lll}
l & k^{\prime} & k \\
l_{1} & k_{1}^{\prime} & k_{1} \\
l_{2} & k_{2}^{\prime} & k_{2}
\end{array}\right\} \\
& \cdot\left[\Phi\left(k_{1} l_{1} k_{1}^{\prime}\right)+(-1)^{l+k_{1}+k_{1}^{\prime}} \Phi\left(k_{2} l_{1} k_{2}^{\prime}\right)\right] .
\end{aligned}
$$

However, the occurrence of the biquadratic term in molecular paramagnetism of specific metal-metal cluster solids suggests that a search for higher order bilinear terms (or $k$-th dot products) in NQR quadrupolar spin-spin effects could be of value.

\section{Symmetry Aspects}

\subsection{Liouvillian Bases for $I \geqq 1$ Spin Clusters and their Symmetry Projection Superoperators}

Irreducible representations, Irreps., of a spin cluster under the symmetry group $\mathrm{S}_{n}$ over Liouville space may be derived from the group algebra by taking the Liouvillian invariance as the product of the $|I M \alpha\rangle$ space invariance of the identical spin system [4]. Such a procedure arises because the $|k q v\rangle$ space constitutes a self inner direct product space under the specific subgroup of identical order to that used in $|I M \alpha\rangle$ space. Hence, it follows that

$$
\mathscr{P}_{\mu}|k q v\rangle=\left\{P_{\mu^{\prime}}|k q v\rangle P_{\mu^{\prime \prime}}^{\dagger}\right\}_{\mu^{\prime} \otimes \mu^{\prime \prime}=\mu}
$$

provides a definition of the projection superoperator. From such considerations, it follows that

$$
\begin{aligned}
& \operatorname{tr} \mathscr{P}_{\mu}|k q v\rangle \\
& \quad=\sum_{\mu^{\prime} \otimes \mu^{\prime \prime}=\mu} \operatorname{tr} P_{\mu^{\prime}}|I M \alpha\rangle \otimes\left(\operatorname{tr} P_{\mu^{\prime \prime}}|I M \alpha\rangle\right)^{\dagger}
\end{aligned}
$$

and that $\mathscr{P}_{\mu}$ and $P_{\mu}(\otimes)$ are related by a simple isomorphism.

The irreps. for the system $[A]_{3}$ for $I=1$ under $\mathrm{S}_{3}$ has been given previously from the invariance properties under the group [4]. Such recent formalisms extend the general forms for $|I M \alpha\rangle$ space due to Flurry and Siddall [5]. Hence the symmetry of Liouville space is defined by

$$
\left[\left\{(\mathrm{SU}(3) \otimes \mathrm{SU}(3) \otimes \mathrm{SU}(3))[x] \mathrm{S}_{3}\right\} \otimes\{\ldots\}^{\dagger}\right] .
$$

Further direct product formation between the irreps. of pairs of interacting spin cluster are common in NMR, but less pertinent for NQR as the magnitude of couplings needs to be larger in order to be detectable. The presence of such coupling can introduce ambiguity in spectral interpretations.

Butler [15] has introduced a convenient notation for sets of states corresponding to spin "particles". This is used in the presentation of the symmetry properties of clusters of $I-3 / 2$ spins under the appropriate $\mathrm{S}_{n}$ group given in Table 1 for $n \leqq 4$. The state space description arises from considering permutation properties of both $3 / 2$ and $1 / 2$ sets of spin functions. The state space irreps. provide suitable generators from which to derive the Liouville space irreps. The series $\mathrm{ICl}_{2}^{+}, \mathrm{ICl}_{3}$ and $\mathrm{ICl}_{4}^{-}$provide, within the $3 / 2$ nuclei, examples of such sets of quadrupolar spin systems under $\mathrm{S}_{n}$. Their $|I M \alpha\rangle$ symmetry is governed by

$$
\left.\left[\{\mathrm{SU}(6)[x] 1\} \otimes\{\mathrm{SU}(4) \otimes \mathrm{SU}(4) \ldots)[x] \mathrm{S}_{n}\right\}\right](15)
$$

in which the clusters of $3 / 2$ spins correspond to 16 , 64 , and 256-fold spaces. The Liouville space total irreps. are derivable from the invariance relations between the two spaces, $\tilde{\chi}_{\mathrm{c}}=\chi_{\mathrm{c}}^{2}$. 
Table 1 . The total and $m$-th irreducible representations, $\Gamma$, for state space arising from the permutation symmetry of the $[A]_{n}, I=3 / 2$, spin cluster under $\mathrm{S}_{2}, \mathrm{~S}_{3}$ and two forms of $\mathrm{S}_{4}$ isomorphic to $\mathrm{D}_{2 \mathrm{~d}}$ and $\mathrm{T}_{\mathrm{d}}$. The numeric representational notation, i.e., $\Gamma=$ (on left of each $\mathrm{S}_{n}$ set) refers to set of states, viz. 3 over set [ $3 \geqq M \geqq-3$ ], analogous to the Butler formalism [15]. The $\tilde{\Gamma}$ formalism is additive over $m$ in recursive sense, $\Gamma^{m}=\Gamma^{(m+1)}+\tilde{\Gamma}^{m}$ originating from $\Gamma^{m(\max )}$.

The respective total irreducible representation, $\Gamma$ and $\Gamma$, over $|I M \alpha\rangle$ and $|k q v\rangle$ spaces are presented at the foot of the table. Notice that the subscripted degeneracies of $\bar{\Gamma}=\mathbf{3}_{(i)}$, etc., do not imply that all such sets belong to one specific representation, only that within such a set the states belong to $\Gamma_{\mu}$.

\begin{tabular}{|c|c|c|c|c|c|c|c|}
\hline $\begin{array}{l}n=2 \\
\bar{\Gamma}\end{array}$ & $\tilde{\Gamma}$ & $\Gamma$ & $\begin{array}{l}n=3 \\
\bar{\Gamma}\end{array}$ & $\tilde{\Gamma}$ & $\begin{array}{l}n=4 ; \\
\bar{\Gamma}\end{array}$ & $\stackrel{\rho_{2 \mathrm{~d}}}{\tilde{\Gamma}}$ & $\begin{array}{l}T_{\mathrm{d}} \\
\tilde{\Gamma}\end{array}$ \\
\hline $\begin{array}{l}3 \\
2 \\
1 \\
0\end{array}$ & $\begin{array}{l}A \\
B \\
A \\
B\end{array}$ & $\begin{array}{l}(A) \\
(A+B) \\
(2 A+B) \\
(2 A+2 B)\end{array}$ & $\begin{array}{l}9 / 2 \\
7 / \mathbf{2}_{(2)} \\
\mathbf{5 / 2}(3) \\
\mathbf{3 / 2} \\
\mathbf{1 / 2} \\
(2)\end{array}$ & $\begin{array}{l}A_{1} \\
E \\
A_{1}+E \\
A_{1}+A_{2}+E \\
E\end{array}$ & $\begin{array}{l}\mathbf{6} \\
\mathbf{5}_{(3)} \\
\mathbf{4}_{(6)} \\
\mathbf{3}_{(10)} \\
\mathbf{2}_{(11)} \\
\mathbf{1}_{(9)} \\
\mathbf{0}_{(4)}\end{array}$ & $\begin{aligned} A_{1} & \\
& B_{2}+E \\
2 A_{1}+B_{1} & +B_{2}+E \\
A_{1}+A_{2} & +2 B_{2}+3 E \\
3 A_{1}+A_{2}+2 B_{1} & +B_{2}+2 E \\
A_{2} & +2 B_{2}+3 E \\
2 A_{1} & +2 B_{1}\end{aligned}$ & 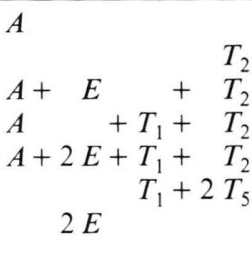 \\
\hline$I M \alpha\rangle$ & & $\begin{array}{l}A+6 B \\
6\end{array}$ & & $\begin{array}{l}20 A_{1}+4 A_{2}+20_{(2)} E \\
=64\end{array}$ & & $\begin{aligned} 55 A_{1} & +15 A_{2}+21 B_{1} \\
& +45 B_{2}+60_{(2)} E\end{aligned}$ & $\begin{aligned} 34 A & +21 E+15 T_{1} \\
& +45 T_{2}=256\end{aligned}$ \\
\hline \multicolumn{3}{|c|}{$\begin{array}{c}k q v \gg ; \\
=256 A+120 B \\
=256\end{array}$} & & \multicolumn{3}{|l|}{$\begin{aligned} 816 A_{1} & +560 A_{2} \\
& +1360_{(2)} E=4096\end{aligned}$} & \\
\hline
\end{tabular}

The symmetry properties over Liouville space of two $[A]_{2}$ systems for higher spins are considered in Table 2 and 3. These are discussed in the context of earlier experimental NQR experiments. Anisotropic $\mathrm{Q}-\mathrm{Q}$ couplings even within a single spin cluster involve multichannel interactions that are analogous to those of the $[\mathrm{AX}]_{n}$ spin system in NMR under $\mathrm{S}_{n}$, discussed by Corio [6] and Jones [17] in state space context.

\subsection{Basis for the solid state $X_{2} Q-Q$ problem}

In the case of solid $\mathrm{Br}_{2}$ both $\mathrm{AX}$ and $[\mathrm{A}]_{2}$ spin systems occur whereas only the latter apply to solid $\mathrm{I}_{2}$. The presence of asymmetry in these systems implies that selective irradiation approach to NQR, such as discussed for the $3 / 2, \eta=0$ problem [18], are not applicable, here. Further, the inclusion of mirror symmetry of the unit cell of such solids necessitates consideration of a pair of such $[A]_{2}$ molecular spin systems. As shown in Tables 2 and 3, the Irreps. for the corresponding $[3 / 2-3 / 2]$ and $[5 / 2-5 / 2]$ systems span

$$
\Gamma=136 A+120 B
$$

and

$$
\Gamma=666 A+630 B
$$

components, respectively, for the single pairs of spins over $k q v\rangle$ space.
To represent the full internal-external symmetrized bases in the multipole formulation, one writes

$Z^{k q}( \pm)_{\mu}=T^{k q}\left(k_{1} k_{2}: \mu\right) \pm T^{k-q}\left(k_{1} k_{2}: \mu\right)$.

An essential point in further detailed treatment of such systems under the impact of anisotropic quadrupolar spin-spin coupling, and including the small e.f.g. asymmetry terms, remains the question of dimensionality. Further, once $\eta$ becomes significant the actual bases required will be linear combinations spanning $Z^{k q}( \pm) \ldots Z^{k q \pm 2}( \pm)$, similar to those of the Asymmetric top [19].

\section{Conclusions}

The use of group theoretical methods with a symmetrized form of hamiltonian facilitates the partitioning of Liouville space into discrete subspaces. The dimensionalities of such subset are minimized under the condition of vanishing e.f.g. asymmetry terms, $\eta_{i}$. The effect of external (crystal) symmetry may be incorporated into the factoring process to optimize the description of multiquantum processes.

\section{Acknowledgements}

This work was carried out with the support of the National Science and Engineering Research Council of Canada. One of us (FT) wishes to acknowledge valuable discussions with Professor H. Haas. 
Table 2. The symmetry-adapted Liouville basis components $T^{k q}(v ; \mu)$ and their respective dimensionalities $n_{\mu}^{q}$ over $|k q v\rangle\rangle$ space for the $[A]_{2}, I_{\mathrm{A}}=3 / 2$ spin problem under $\mathrm{S}_{2}$. The lower set of components $(\dagger)$ have been presented in detail in Table 3 of $[4$ a]. Both Table 2 and Table 3 arise from the total Liouville basis set being governed by

$$
\left\{\prod_{i}^{2}(\otimes \mathrm{SU}(2 I+1))_{i}[x] \mathrm{S}_{2}\right\}\left\{\prod_{i^{\prime}}^{2}(\otimes \mathrm{SU}(2 I+1))_{i^{\prime}}[x] \mathrm{S}_{2}\right\}^{\dagger}
$$

for this type of general $I$ spin system

\begin{tabular}{lllr}
\hline$(3 / 2: 3 / 2), \mathrm{S}_{2} \Leftrightarrow \mathrm{C}_{2}$ & \\
\hline$k q v\rangle$ & $n_{\mathscr{d}}$ & $n_{\mathscr{B}}$ \\
\hline$T^{6 q}(33)$ & & $T^{5 q}(33)$ & \\
$T^{4 q}(33)$ & & $T^{3 q}(33)$ & \\
$T^{2 q}(33)$ & & $T^{1 q}(33)$ & \\
$T^{00}(33)$ & & & 21 \\
$T^{k q}(32: \mathscr{A})$ & 35 & $T^{k q}(32: \mathscr{B})$ & 35 \\
$T^{k q}(31: \mathscr{A})$ & 21 & $T^{k q}(31: \mathscr{B})$ & 21 \\
$T^{k q}(30: \mathscr{A})$ & 7 & $T^{k q}(30: \mathscr{B})$ & 7 \\
\hline & & & 84 \\
$\lceil\dagger\rceil$ & 91 & {$[\dagger]$} & 36 \\
\hline$\sum_{k, k_{1} k_{2}} n_{\mu}$ & 136 & & \\
\hline
\end{tabular}

[1] T. H. Siddall and R. L. Flurry, J. Magn. Reson. 39, 487 (1980).

[2] S. Grechinshkin and N. E. Ainbinder, J.E.T. Phys. 28, 407 (1968).

[3] B. C. Sanctuary, J. Magn. Reson. 61, 116 (1985).

[4] (a) B. C. Sanctuary and F. P. Temme, Molec. Phys. 55, 1049 (1985); (b) F. P. Temme and B. C. Sanctuary, ibid. (submitted).

[5] R. L. Flury and T. H. Siddall, in: Advances in Group Theory and Its Physical Applications (J. C. Donini, ed.). Plenum Press, 1979.

[6] P. L. Corio, The Structure of NMR Spectra. Academic Press, New York 1967.

[7] S. Kojima and K. Tsukada, J. Phys. Japan 10, 591 (1955). - T. Itoh and K. Kambe, ibid. 12, 763 (1957).

[8] T. J. Bastow, S. N. Stuart, and I. D. Campbell, J. Molec. Struct. 111, 71 (1983).

[9] H. Barfuss and B. Roesler, ibid. 111, 21 (1983).

[10] J. S. Griffiths, Struct. Bonding Berlin 10, 87 (1972).
Table 3. The symmetry-adapted Liouville basis components $T^{k q}(v ; \mu)$ and their respective dimensionalities $n_{\mu}^{q}$ for the $[A]_{2}, I_{\mathrm{A}}=5 / 2$ spin system under $\mathrm{S}_{2}$.

\begin{tabular}{lrlr}
\hline$(5 / 2: 5 / 2), \mathrm{S}_{2}$ & & & \\
\hline$k q v\rangle$ & $n_{\mathscr{A}}$ & & $n_{\mathscr{B}}$ \\
\hline$T^{10 q}(55)$ & 21 & & \\
$T^{8 q}(55)$ & 17 & $T^{9 q}(55)$ & 19 \\
$T^{6 q}(55)$ & 13 & $T^{7 q}(55)$ & 15 \\
$T^{4 q}(55)$ & 9 & $T^{5 q}(55)$ & 11 \\
$T^{2 q}(55)$ & 5 & $T^{3 q}(55)$ & 7 \\
$T^{00}(55)$ & 1 & $T^{1 q}(55)$ & 3 \\
& 66 & & 55 \\
$T^{\text {even } k, q(44)}$ & 45 & $T^{\text {odd } k, q}(44)$ & 36 \\
$T^{k q}(54: \mathscr{A})$ & & $T^{k q}(54: \mathscr{B})$ & \\
$T^{k q}(50: \mathscr{A})$ & & $T^{k q}(50: \mathscr{B})$ & \\
$T^{k q}(43: \mathscr{A})$ & & $T^{k q}(43: \mathscr{B})$ & \\
$T^{k q}(40: \mathscr{A})$ & 419 & $T^{k q}(40: \mathscr{B})$ & 419 \\
{$[$ Table 3] } & 136 & \\
$\Gamma_{\mu}$ & 666 & \\
\hline$\sum \Gamma_{\mu}$ & 1296 & \\
\hline$\mu$
\end{tabular}

[11] W. E. Hatfield, in: Theory and Application of Molecular Paramagnetism (E. Bordeaux, ed.). Wiley, New York 1976.

[12] B. C. Sanctuary, P. Osment, and H. K. Halstead, Molec. Phys. 49, 753 (1983).

[13] M. S. Krishnan and B. C. Sanctuary, Z. Naturforsch. 41 a, 353 (1986).

[14] B. C. Sanctuary and L. Selwyn, J. Chem. Phys. 74, 906 (1981).

[15] P. H. Butler, Point Group Symmetry Applications. Plenum Press, New York 1979.

[16] B. C. Sanctuary, Molec. Phys. 55, 1017 (1985).

[17] R. G. Jones, in: Progress and Principles of NMR, Vol. 1, Chapt. II, 1969.

[18] B. C. Sanctuary and F. P. Temme, J. Molec. Struct. 111, 71 (1983).

[19] M. Tinkham, Group Theory and Quantum Mechanics, McGraw Hill, New York 1964. 